\title{
Ophthalmic pathologies in an older HIV-positive Tanzanian population and their association with neurological morbidity and evaluation of a low- resource screening strategy
}

Grace George ( $\sim$ grace.george@newcastle.ac.uk)

Newcastle University Faculty of Medical Sciences https://orcid.org/0000-0002-4717-111X

Declan C Murphy

Newcastle University

HD Jeffry Hogg

Newcastle Upon Tyne Hospitals NHS Trust: Newcastle Upon Tyne Hospitals NHS Foundation Trust Japhet Bright Boniface

Mawenzi Regional Referral Hospital

\section{Sarah Urasa}

KCMU College: Kilimanjaro Christian Medical University College

Justus Rwiza

KCMU College: Kilimanjaro Christian Medical University College

Livin Uwemeye

KCMU College: Kilimanjaro Christian Medical University College

Clare Bristow

Newcastle University

Grace Hillsmith

Newcastle University

\section{Emma Rainey}

Newcastle University

Richard Walker

Newcastle University

\section{William K Gray}

Northumbria Healthcare NHS Foundation Trust

\section{Stella Maria-Paddick}

Newcastle University

\section{Research}

Keywords: Eye disease, HIV

Posted Date: April 8th, 2021 
DOl: https://doi.org/10.21203/rs.3.rs-398160/v1

License: (c) This work is licensed under a Creative Commons Attribution 4.0 International License. Read Full License 


\section{Abstract \\ Background}

Globally, 43 million people are living with HIV, 90\% in developing countries. Increasing life expectancy with combination antiretroviral therapy (CART) results in chronic complications, including HIV-associated neurocognitive disorders (HAND) and eye diseases. HAND screening is currently challenging.

\section{Objectives}

To evaluate clinical utility of HIV-retinopathy as a screening measure of HAND in older cART-treated individuals in Tanzania and feasibility of smartphone-based retinal screening in this low-resource setting.

\section{Methods}

A cross-sectional systematic sample aged $\geq 50$-years attending routine HIV follow-up in Tanzania were comprehensively assessed for HAND by American Academy of Neurology criteria and received ophthalmic assessment including smartphone-based retinal imaging. HAND and ophthalmic assessments were independent and blinded. Diagnostic accuracy was evaluated by AUROC curves.

\section{Results}

Of 129 individuals assessed, $69.8 \%$ were visually impairment. Six had suspected HIV retinopathy. HAND prevalence was $66.7 \%$. HIV retinopathy was significantly associated with HAND but HIV-disease factors (CD4, viral load) were not. Diagnostic accuracy of HIV-retinopathy for HAND was poor (AUROC 0.545-0.617) but specificity and positive predictive value were high.

\section{Conclusions}

Ocular pathology and HAND appear highly prevalent in this low-resource setting. Although retinal screening cannot be used alone identify HAND, prioritization of individuals with abnormal retinal screening is a potential strategy in low-resource settings.

\section{Background}

Almost 43 million people are infected by the human immunodeficiency virus (HIV) globally, with $90 \%$ of cases in developing countries[1]. In sub-Saharan Africa (SSA), almost 25 million individuals currently live with HIV[2]. Increasing provision of combination antiretroviral therapy (CART) is resulting in both improving life expectancy and emergence of chronic HIV-related complications and chronic diseases as previously seen in high-income countries. HIV-associated neurocognitive disorders (HAND) affect up to $50 \%$ of people treated for HIV and are associated with increased morbidity and mortality [3, 4]. HAND are classified by severity: asymptomatic neurocognitive impairment (ANI); mild neurocognitive disorder (MND) and; HIV-associated dementia (HAD)[5]. 
Early identification of HAND and subsequent prompt initiation of treatment, prior to progression of HAND stage, may reduce mortality and reverse neurocognitive deficits[3]. Screening for, and identification of HAND are currently challenging, in SSA and elsewhere. Difficulties include shortage of neurology and psychiatry specialist services in SSA, lack of accurate brief screening measures[6] and current consensus diagnostic criteria requiring detailed neuropsychological assessment, impractical in busy clinical settings. Additionally, the use of mobile technologies to help address health and human resource shortages in low-resource settings is a recent and expanding area of research and has previously been evaluated for use in remote grading of ophthalmic disease in SSA[7].

HIV-associated eye diseases occur in up to $70 \%$ of HIV positive individuals[8]. Early recognition and treatment can prevent or cure $80 \%$ of cases[9]. Features observed in the retina may provide insight about the systemic health of individuals[10], such as in previous studies where ophthalmoscopy identified biomarkers of hypertension and its severity[11]. Vascular diseases are also common amongst older people living with HIV, and are often undertreated in SSA[12]. There appears to be a strong association between HIV and vasculopathies such as stroke[13], and renal disease[14] and retinal disease[15]. Retinal vascular changes has been shown to positively correlate with white matter microstructural changes observed using magnetic resonance imaging in non-HIV positive patients[10]. As white matter disease is also seen in both treated and untreated HIV cases and is associated with $\operatorname{HAND}[16,17]$, we hypothesized that retinal imaging could help identify individuals at risk of HAND.

In this study we aimed to determine the clinical utility of HIV-retinopathy as a potential predictive biomarker of HIV-associated neurological complications in older cART-treated HIV positive individuals in Tanzania. A second objective was to determine if retinopathy could be a potential alternative to detailed neuropsychological assessment in a resource poor setting. In addition, we aimed to determine if remote retinal screening using a smartphone based retinal camera was a potentially useful strategy in this context.

\section{Methods}

A cross-sectional study at a single government-funded HIV clinic in Northern Tanzania. Participant selection Participants were recruited as part of a larger cross-sectional study of HAND and underwent detailed neurocognitive and clinical assessment. Criteria for inclusion were: $\geq 50$-years of age, HIV-positive, attending routine follow up and not acutely unwell. A systematic sampling technique was employed with every second or third eligible participant approached for inclusion depending on daily capacity. All of those recruited within the larger study were invited to attend an additional appointment for ophthalmoscopic examination. Informed consent The purpose for the study and their specific involvement was explained to each potential participant and written informed consent was obtained. Where capacity was in doubt, consent was obtained from close relatives. Ethical approval was obtained from the National Institute for Medical Research (NIMR/HQ/R.8a/Vol. IX/21.36) and Kilimanjaro Christian Medical University College Research Ethics Committee (n.896). This study abides by the tenets of the declaration of Helsinki. Assessment Baseline sociodemographic data of participant, plus HIV disease severity data from standard data sheets, were collected (Appendix 1). A set of functional assessments were used, including Karnofsky Performance Status[18], Rockwood Clinical Frailty Scale[19], and Intervention for Dementia in Elderly Africans (IDEA) low-literacy brief cognitive screen, previously validated for dementia in SSA[20]. Hypertensive and diabetic status were recorded. Visual acuity (Va) was assessed with

Page $4 / 15$ 
available correction using the Landolt broken-ring low-literacy " $\mathrm{C}$ " chart with notations for testing at 3 meters due to unknown rates of illiteracy amongst the cohort. Visual fields were tested using confrontation (quadrant finger counting) and color vision with Ishihara charts. All participants underwent direct ophthalmoscopic examination following pupil dilatation (1\% tropicamide) and retinal images were obtained using the iNview retinal camera (VOLK, Cleveland, USA). Images were independently reviewed by 3 ophthalmologists, 2 of whom were based in Tanzania (JR, LU) and 1 from the UK (JH). HAND assessment and diagnosis HAND were diagnosed by consensus using standard criteria[5] based on previously-obtained local normative values for neuropsychological battery measures, clinical/neurological examination findings and a collateral history from a close relative or friend. All participants underwent a detailed low-literacy neuropsychological assessment battery, the details of which have previously been published[6]. Domains assessed included working memory, verbal memory (learning, delayed recall and recognition memory), fine motor control, motor speed, visuoconstruction, executive function and comprehension. Ophthalmologist criteria for defining eye disease The definitions for visual impairment used followed those given in the National Sensory Impairment Partnership's Framework[21]. Mild-moderate visual impairment was defined as LogMAR 0.30.8. "glaucoma suspect" was termed if cup-to-disc ratio was $>0.6[22]$. A Tanzania-based ophthalmology trainee (JR) determined whether glaucoma could be diagnosed based on ICD-10 criteria[22]. HIV retinopathy was assumed if any of the following features were observed upon ophthalmoscopy: retinal hemorrhages; microaneurysms; areas of capillary non-perfusion; or cotton wool spots. Statistical analysis All statistical analyses were performed using IBM SPSS software V24.0. Normally distributed data were described using mean, standard deviation (SD) and 95\% confidence intervals (Cl). Comparisons were made using independent t-tests. Nonnormally distributed data were described using median, interquartile range (IQR) and frequency and compared using Mann-Whitney $\mathrm{U}$ test. Categorical variables were described using frequency and compared using chisquared test and with the Fisher's exact test correction where appropriate. Logistic regression was used to evaluate potential relationships between outcomes and explanatory covariates. Statistical significance was defined as $P \leq 0.05$. The performance of the smartphone HIV retinopathy screen was investigated using Area Under the Receiver Operating Characteristic (AUROC) curve analysis. Diagnostic accuracy was analyzed for HAND and symptomatic HAND (s-HAND, MND/HAD). To determine diagnostic accuracy for HAND, ANI/MND/HAD were coded 1 and all others coded 0 . For S-HAND, MND/HAD were coded 1 , and all others 0 . Diagnostic accuracy was also analyzed for HIV stage 3 or 4 coded as 1 and all others coded 0 as well as analyzed for detectable viral load coded as 1 and undetectable as 0 . When grouped together, the predictive value of HIV retinopathy to determine late HIV stage, detectable viral load as well as HAND and s-HAND was analyzed.

\section{Results}

\section{Population characteristics}

Of the 762 individuals aged $\geq 50$ years attending follow-up during the study period, 145 were systematically sampled and consented to inclusion. Of these, 129 completed the ophthalmologic assessment and had complete data (Figure 1). There was no significant difference in age, CD4 count or viral load between those who had complete data and those who did not. 
Median patient age was 56 years (IQR: 53 to 61), 83 (64.3\%) were female, $88(68.2 \%)$ had at least 5-7 years of primary-school education and 8 (6.2\%) were illiterate. Median time since HIV diagnosis was 10.5 years (Range: 3 months to 23 years) and median nadir CD 4 count was 168 cells $/ \mathrm{mm}^{3}$ (IQR: 106-251). All but one participant was receiving antiretroviral treatment when enrolled in the study. HIV-disease appeared well managed with current median CD4 434 cells/ $\mathrm{mm}^{3}$ and $76 \%$ with suppressed HIV viral load (defined as <20 copies/ml). Twenty-one (16.3\%) reported a prior hypertension diagnosis, 11 (52.4\%) of whom were on anti-hypertensives. Thirty-eight (29.5\%) of patients were hypertensive in clinic (blood pressure (BP) >140/90 millimeter of mercury $(\mathrm{mmHg})$. The mean BP in clinic was $134.5 / 81.2 \mathrm{mmHg}$ with a maximum systolic of $219 \mathrm{mmHg}$. Seven (5.4\%) patients reported being diabetic, $5(71.4 \%)$ of whom were on treatment.

Forty-three patients (33.3\%) did not meet HAND criteria, 41 (31.8\%) met criteria for ANI, 43 (33.3\%) met criteria for MND and 2 patients (1.6\%) were diagnosed with HAD.

\section{Overall Prevalence of Ophthalmic Disease}

Recent visual changes were self-reported by 71 patients (55\%), but none reported having sought ophthalmological review.

Fifty-three patients $(41.1 \%, 95 \% \mathrm{Cl}: 32.6-49.6)$ had mild-moderate visual impairment $(0.3<\mathrm{Va} \leq 0.8)$ and 37 (28.7\%, 95\% Cl: 20.9-36.5) severe visual impairment (Va>0.8). Six (4.7\%) had a visual field defect, 4 (3.1\%) cataracts and 5 (3.9\%) abnormal color vision. Fifty-eight patients (45\%) had Va>0.6 and abnormal fundal signs were seen in 53 patients (41.1\%), including 16 (12.4\%) referred for suspected glaucoma and 6 (4.7\%) for suspected HIV retinopathy. One showed signs consistent with cytomegalovirus retinitis. Once reviewed by an ophthalmology specialist (JH), HIV retinopathy was suspected in 13 patients (10.1\%) and glaucoma was suspected in 19 patients (14.7\%) (CDR>0.6) but following diagnostic assessments only one patient was diagnosed with bilateral primary angle closure glaucoma. Sixty-seven patients $(51.9 \%)$ were referred for full ophthalmic assessment.

\section{$\underline{\text { HIV Retinopathy and its association with HAND }}$}

HIV retinopathy features were significantly associated with both HAND diagnosis and HAND severity (Table 1). HIV retinopathy was associated with patient-reported vision loss during the previous 3 months $\left(\chi^{2}(1, n=129)\right.$ : 5.111; $p=0.037)$.

Table 1 - Comparison of characteristics between patients with suspected human immunodeficiency virus retinopathy and those without 


\begin{tabular}{|c|c|c|c|c|}
\hline \multicolumn{2}{|l|}{ Variable } & $\begin{array}{l}\text { Patients with HIV } \\
\text { Retinopathy }\end{array}$ & $\begin{array}{l}\text { Patients without HIV } \\
\text { retinopathy }\end{array}$ & Significance \\
\hline \multicolumn{2}{|l|}{ Age (median) } & 60 & 56 & $U=612.5$ \\
\hline \multirow{2}{*}{\multicolumn{2}{|c|}{$\mathrm{N}=129$}} & & & $Z=-1.309$ \\
\hline & & & & $p=0.191$ \\
\hline \multicolumn{2}{|l|}{ Gender in \% (F:M) } & $7: 6$ & $76: 40$ & $X^{2}=0.694$ \\
\hline \multicolumn{2}{|l|}{$N=129$} & & & $\mathrm{p}=0.543$ \\
\hline \multirow{3}{*}{\multicolumn{2}{|c|}{$\begin{array}{l}\text { Median CD4 count } \\
\mathrm{N}=114\end{array}$}} & 474 & 434 & $U=558.0$ \\
\hline & & & & $Z=-0.088$ \\
\hline & & & & $p=0.930$ \\
\hline \multirow{3}{*}{\multicolumn{2}{|c|}{$\begin{array}{l}\text { Detectable viral load (frequency) } \\
\mathrm{N}=105\end{array}$}} & Detectable: 5 (4.8\%) & Detectable: 39 (37.1\%) & $x^{2}=0.298$ \\
\hline & & Non detectable: 5 & Non-detectable: 56 & $\mathrm{p}=0.739$ \\
\hline & & $(4.8 \%)$ & & \\
\hline \multirow{2}{*}{\multicolumn{2}{|c|}{$\begin{array}{l}\text { Karnofsky Performance Status } \\
\text { (median) }\end{array}$}} & 90 & 100 & $U=555.0$ \\
\hline & & & & $Z=-1.787$ \\
\hline \multicolumn{2}{|l|}{$N=129$} & & & $\mathrm{p}=0.074$ \\
\hline \multicolumn{2}{|c|}{ Clinical Frailty Scale (median) } & 2.00 & 2.00 & $U=558.5$ \\
\hline \multirow{2}{*}{\multicolumn{2}{|c|}{$N=129$}} & & & $Z=-1.727$ \\
\hline & & & & $p=0.084$ \\
\hline $\begin{array}{l}\text { HAND diagnoses } \\
\text { (frequency) }\end{array}$ & $\begin{array}{l}\text { No } \\
\text { HAND }\end{array}$ & $2(15.4 \%)$ & $41(31.8 \%)$ & \multirow{4}{*}{$\begin{array}{l}U=510.0 \\
Z=-2.051 \\
p=0.04\end{array}$} \\
\hline \multirow[t]{3}{*}{$N=129$} & ANI & $3(23.1 \%)$ & $38(29.5 \%)$ & \\
\hline & MND & $7(53.8 \%)$ & $36(27.9 \%)$ & \\
\hline & HAD & 1 (7.7\%) & $1(0.8 \%)$ & \\
\hline \multicolumn{2}{|c|}{ Subjective change in vision $(\mathrm{Y}: \mathrm{N})$} & $11: 2$ & $60: 56$ & $x^{2}=5.111$ \\
\hline \multicolumn{2}{|l|}{$\mathrm{N}=129$} & & & $p=0.037$ \\
\hline
\end{tabular}

Using logistic regression, HAND severity and self-reported visual change were assessed for significance against HIV retinopathy. The significance intervals, odds ratios and confidence intervals are shown in Table 2. This shows HAND severity is associated with HIV retinopathy, adjusting for co-variates.

Table 2 - Summary of regression analysis 


\begin{tabular}{|llll|}
\hline Variable & Significance & Odds Ratio & $95 \% \mathrm{Cl}$ \\
\hline HAND severity & $0.025^{\star}$ & 2.406 & $1.117-5.184$ \\
\hline Change in Vision & 0.061 & 0.216 & $0.044-1.075$ \\
\hline
\end{tabular}

Diagnostic Accuracy

The predictive value of HIV retinopathy for HAND, s-HAND, late HIV stage and detectable viral load are presented in Table 2. Overall diagnostic accuracy was poor. AUROC ranged from $0.545-0.617$, with $95 \% \mathrm{Cl}$ spanning 0.5 for each variable. Specificity was high throughout whereas sensitivity was low. Positive predictive value (PPV) was high for HAND and HIV stage 3 whereas PPV for s-HAND and detectable VL was low. Of all the screening options evaluated, the highest screening accuracy was achieved for HAND using HIV retinopathy screen (AUROC $=0.617$, sensitivity $13.4 \%$, specificity $95.7 \%$ and PPV 0.846 ).

Table 3 - AUROC for relation of variables to HIV retinopathy

\begin{tabular}{|llllll|}
\hline & $\begin{array}{l}\text { AUROC } \\
(95 \% \text { Cl) }\end{array}$ & Sensitivity & Specificity & $\begin{array}{l}\text { Positive } \\
\text { Predictive Value }\end{array}$ & $\begin{array}{l}\text { Negative } \\
\text { Predictive value }\end{array}$ \\
\hline HAND (n=82) & $\begin{array}{l}0.617 \\
(0.471-\end{array}$ & 0.134 & 0.957 & 0.846 & 0.388 \\
\hline $\begin{array}{l}\text { Symptomatic HAND } \\
(\mathrm{n}=27)\end{array}$ & $0.763)$ & & & & \\
$(\mathrm{MND} / \mathrm{HAD})$ & $\begin{array}{l}(0.424- \\
0.771)\end{array}$ & 0.185 & 0.922 & 0.385 & 0.81 \\
$\begin{array}{l}\text { HIV Stage 3/4 } \\
(\mathrm{n}=105)\end{array}$ & 0.557 & 0.144 & 0.957 & 0.923 & 0.191 \\
& $\begin{array}{l}(0.404- \\
0.711)\end{array}$ & & & & \\
\hline Detectable VL $(\mathrm{n}=44)$ & 0.545 & 0.144 & 0.918 & 0.5 & 0.589 \\
& $\begin{array}{l}(0.355- \\
0.734)\end{array}$ & & & \\
\hline
\end{tabular}

\section{Discussion}

Our findings suggest that HIV retinopathy may be associated with HAND in older adults receiving long term HIV treatment in this setting. However, identification of HIV retinopathy does not appear to be a useful strategy for identifying those with HAND, or those with frailty. AUROC was not performed on clinical frailty scale as there was no statistically significant association with HIV retinopathy. The high specificity of HIV retinopathy screening may have implications for clinical practice because retinal imaging may show potential to 'rule in' which individuals with HIV are likely to be at risk of cognitive impairment. Those negative for HIV retinopathy, have a higher probability of not meeting HAND criteria (normal cognition). In addition, a negative HIV retinopathy assessment does not reliably exclude HAND. This means that HIV retinopathy screening cannot be 
used alone to determine who might need assessment for HAND. However, retinal screening could allow staff to prioritize individuals with retinal signs, due to high PPV.

Although the relationship between HIV retinopathy and HAND is unclear, there are commonalities in the pathogeneses of both HIV-related complications which may explain their relationship. HIV retinopathy most likely occurs secondary to microvasculopathy from either immune complex deposition, increased plasma viscosity or vascular endothelium invasion by HIV, opportunistic infections or malignancy[23]. HAND occurs through a number of mechanisms including opportunistic central nervous system (CNS) infections, direct neurotoxic effect of the HIV virus and neurotoxic effect of cART[4]. Contributory factors include HIV disease severity, accelerated vascular disease and frequent co-morbidities[4].

The World Health Organization (WHO) advocates that screening for mental disorders be integrated into chronic disease monitoring to address the shortage of specialist personnel in low-resource settings[24]. Eye disease is currently a major cause of morbidity and disability in both HIV and non-HIV populations and disproportionately affects older people[25]. Cataracts, glaucoma, uncorrected refractive error and trauma are the major reported causes of visual impairment[25]. Identification and screening for ophthalmic disease in low-resource settings is currently challenged by similar issues to those noted for chronic complications of HIV. These include lack of specialist personnel, particularly in rural and remote areas, a focus on acute intervention rather than primary care and prevention, lack of integrated referral systems and access to affordable necessary equipment[26,27].

Using mobile technologies to help address health and human resource shortages in low-resource settings is a recent and expanding area of research and is increasingly feasible given the rapid increase in smartphone use across SSA[28]. Low-cost smartphone applications have been evaluated for use in remote grading of ophthalmic disease in SSA[7]. Non-clinical imagers were able to capture images at a standard that enabled remote grading at the level of a desktop retinal camera[7]. In some regions of SSA there is only one ophthalmologist per 2.5 million people, so remote retinal imaging could ameliorate the impact of understaffing[29].

In this study, we demonstrate a high prevalence of visual impairment in our cohort of HIV-positive patients, with $41.1 \%$ having mild-moderate visual impairment and $28.7 \%$ severe visual impairment. A previously published study reported that $11.2 \%$ of HIV-positive individuals aged 18 years or greater had a visual acuity of 0.2 or more in at least one eye[30]. One previous study in HIV-negative individuals aged 50 or greater report visual impairment of $13.6 \%$ in SSA[31]. Our prevalence was much higher. This difference may relate to our cohort being 50 years or older whereas other studies investigated younger cohorts. In addition, other explanations may include a risk of false positives from unidentified refractive causes of acuity loss, a lower threshold for diagnosis of visual impairment or our small sample size[30]. Factors relating to the willingness of patients to present with ophthalmic features may also contribute, perhaps due to lack of symptom knowledge, cost or impaired cognition. Since there is a high prevalence of eye disease in this cohort, it is prudent to screen for it.

We suspect the high prevalence of visual impairment may be seen also in the background local population, due to lack of routine eye tests and low patient presentation, even when high quality, affordable services are available[32]. Rates of visual impairment in the remote and rural areas may in fact be worse than those regularly attending hospital due to cost and availability of transport. In addition, low-cost retinal imaging may enable the effective monitoring of patients with HIV-associated ophthalmic diseases and could address 
challenges in healthcare provision relating to the limited availability of healthcare professionals in these regions[29].

Strengths and Limitations of the study.

To our knowledge, this is the only study on HIV retinopathy in Tanzania, thus providing unique data on visual impairment and ocular disease prevalence. Previous studies looking at disease amongst HIV-infected individuals in SSA, excluded patients with confusion or altered mental state[30]. Since this study was able to assess the link between HIV ophthalmic diseases and HAND, this was an additional strength.

There are several limitations to this study. Images obtained from VOLK iNview retinal camera were of low-tomoderate quality so subtle changes or more complex pathologies may have been missed. Therefore, there is a substantial reliance on external referrals to ensure a correct diagnosis is made.

There are non-HIV causes of the fundal signs used to define suspected HIV retinopathy, such as hypertensive and diabetic retinopathy. A large proportion of our cohort had a blood pressure over $140 \mathrm{mmHg}$, however 'whitecoat effect' is relatively common in SSA[33]. Seven of the 13 (53.8\%) suspected HIV retinopathy patients had hypertension in clinic; we cannot exclude hypertensive retinopathy in these individuals. Despite individuals not self-reporting as diabetic and being under regular clinic follow-up, we found that 9 patients had glycosuria, a clinical sign of undiagnosed or untreated diabetes. 5 of these 9 had retinal signs thought to be consistent with HIV retinopathy but without measuring HbA1c, we cannot exclude diabetic retinopathy in these individuals.

This research only relates to HIV positive adults aged 50-years or older. This may be a strength in that studies of this older CART-treated population are few in SSA, but limits generalizability.

\section{Conclusions And Recommendations For Future Work}

Ocular pathology and HAND were both common in this patient population. We demonstrate that HIV retinopathy is associated with the presence of HAND. This has implications for clinical practice as retinal imaging may have 'rule in' value in predicting which individuals with HIV are likely to be at risk of cognitive impairment. Substantial clinical need for low-resource screening tools for HAND has been identified and although retinal screening cannot be used alone to determine who might need assessment for HAND, it may offer healthcare professionals a tool to enable patient prioritization.

As early diagnosis of ocular pathology is crucial to allow for timely intervention, portable and affordable tools which image the retina and enable remote diagnosis and monitoring of ophthalmic conditions in HIV-positive individuals. As technology develops, such assessments will become more accurate and useful.

\section{Abbreviations}

ANI - Asymptomatic neurocognitive impairment

AUROC - Area Under Receiver Operating Characteristic

$\mathrm{BP}$ - blood pressure

Page 10/15 
$\mathrm{Cl}$ - confidence intervals

CNS - central nervous system

HAD - HIV-associated dementia

HAND - HIV-associated neurocognitive disorder

HIV - Human Immunodeficiency Virus

IDEA - Intervention for Dementia in Elderly Africans

IQR - interquartile range

$\mathrm{mmHg}$ - millimeters of mercury

MND - mild neurocognitive disorder

PPV - positive predictive value

SD - standard deviations

SSA - Sub-Saharan Africa

s-HAND - symptomatic HAND

$\mathrm{Va}$ - visual acuity

WHO - world health organization

\section{Declarations}

Ethics approval and consent to participate: The purpose for the study and their specific involvement was explained to each potential participant and written informed consent was obtained. Where capacity was in doubt, consent was obtained from close relatives. Ethical approval was obtained from the National Institute for Medical Research (NIMR/HQ/R.8a/Vol. IX/21.36) and Kilimanjaro Christian Medical University College Research Ethics Committee (n.896).

Consent for publication: not applicable

Availability of data and materials: The datasets used and/or analysed during the current study are available from the corresponding author on reasonable request.

Competing interests: The authors declare that they have no competing interests

Funding: This work was supported by an Academy of Medical Sciences (AMS) start-up grant awarded to Dr Paddick. 
Authors' contributions: GG prepared the literature review, completed the study and wrote the paper. DM helped write the paper. HJH analyzed the retinal imaging and therefore helped to complete the study. JB, SU, JR, LU, $\mathrm{CB}, \mathrm{GH}$ and ER helped in the set up and running of the study. RW, WG and SMP helped with study design, directed its implementation as well as helped write up the study.

Acknowledgements: We thank research assistants and coordinators: Dr Sengua Koipapi, Dr Jane Rogathi, Mr Aloyce Kisoli, Mr Dennis Clement, Ms Cecilia Chuwa, Ms Happiness Mosha alongside all staff, patients and carers attending Mawenzi Regional Referral Hospital HIV care and treatment center and the MRRH hospital management for accommodating and hosting the study.

\section{References}

1. World Health Organization, UNAIDS, UNICEF. Global hiv/aids response: epidemic update and health sector progress towards universal access: progress report. Published online 2011:224.

2. Kharsany ABM, Karim QA. HIV Infection and AIDS in Sub-Saharan Africa: Current Status, Challenges and Opportunities. The open AIDS journal. 2016;10:34-48. doi:10.2174/1874613601610010034

3. Saylor D, Nakigozi G, Nakasujja N, et al. HIV-associated Neurocognitive Disorder leads to Death. In: HAND: BIOMARKERS, RISKS, PREVALENCE. ; 2019:99733. https://2jg4quetidw2blbbq2ixwziw-wpengine.netdnassl.com/wp-content/uploads/sites/2/posters/2019/1430_Sacktor_0425.pdf

4. Clifford DB, Ances BM. HIV-associated neurocognitive disorder. The Lancet Infectious Diseases. Published online 2013. doi:10.1016/S1473-3099(13)70269-X

5. Antinori A, Arendt G, Becker JT, et al. Updated research nosology for HIV-associated neurocognitive disorders. Neurology. 2007;69(18):1789-1799. doi:10.1212/01.WNL.0000287431.88658.8b

6. Kellett-Wright J, Flatt A, Eaton P, et al. Screening for HIV-Associated Neurocognitive Disorder (HAND) in Adults Aged 50 and Over Attending a Government HIV Clinic in Kilimanjaro, Tanzania. Comparison of the International HIV Dementia Scale (IHDS) and IDEA Six Item Dementia Screen. AIDS and Behavior. 2020; (0123456789). doi:10.1007/s10461-020-02998-9

7. Bastawrous A, Giardini ME, Bolster NM, et al. Clinical validation of a smartphone-based adapter for optic disc imaging in Kenya. JAMA Ophthalmology. 2016;134(12):151-158.

doi:10.1001/jamaophthalmol.2015.4625

8. Chiotan C, Radu L, Serban R, Cornăcel C, Cioboată M, Anghelie A. Posterior segment ocular manifestations of HIV/AIDS patients. Journal of medicine and life. 2014;7(3):399-402.

http://www.ncbi.nlm.nih.gov/pubmed/25408764\%0Ahttp://www.pubmedcentral.nih.gov/articlerender.fcgi? artid=PMC4233448

9. World Health Organization. Blindness and visual impairment. Published 2018. Accessed July 3, 2019. https://www.who.int/news-room/fact-sheets/detail/blindness-and-visual-impairment

10. Mutlu U, Cremers LGM, de Groot M, et al. Retinal microvasculature and white matter microstructure: The Rotterdam Study. Neurology. 2016;87(10):1003-1010. doi:10.1212/WNL.0000000000003080

11. Jones R, Putnam HWI, Philippin $\mathrm{H}$, et al. Retinal imaging to identify target organ damage in older Africans: A pilot study. Journal of clinical hypertension (Greenwich, Conn). 2018;20(9):1296-1301.

doi:10.1111/jch.13352

Page 12/15 
12. Benjamin LA, Corbett EL, Connor MD, et al. HIV, antiretroviral treatment, hypertension, and stroke in Malawian adults. Neurology. 2016;86(4):324-333. doi:10.1212/WNL.0000000000002278

13. Walker RW, Jusabani A, Aris E, et al. Stroke risk factors in an incident population in urban and rural Tanzania: A prospective, community-based, case-control study. The Lancet Global Health. 2013;1(5):282285. doi:10.1016/S2214-109X(13)70068-8

14. Naftalin C, Nathan B, Hamzah L, Post FA. HIV-associated kidney disease in the context of an aging population. Sexual Health. 2011;8(4):485-492. doi:10.1071/sh10146

15. Lima B. Ophthalmic Manifestations of HIV Infection. Digital Journal of Ophthalmology. 2004;10(3). Accessed June 10, 2019. http://www.djo.harvard.edu/site.php?url=/physicians/oa/674

16. Hoare J, Fouche J-P, Phillips N, et al. Clinical associations of white matter damage in cART-treated HIVpositive children in South Africa. Journal of NeuroVirology. 2015;21(2):120-128. doi:10.1007/s13365-0140311-1

17. Alakkas A, Ellis RJ, Watson CWM, et al. White matter damage, neuroinflammation, and neuronal integrity in HAND. Journal of NeuroVirology. 2019;25(1):32-41. doi:10.1007/s13365-018-0682-9

18. Sperduto PW, Kased N, Roberge D, et al. Summary report on the graded prognostic assessment: An accurate and facile diagnosis-specific tool to estimate survival for patients with brain metastases. Journal of Clinical Oncology. 2012;30(4):419-425. doi:10.1200/JC0.2011.38.0527

19. Church S, Rogers E, Rockwood K, Theou O. A scoping review of the Clinical Frailty Scale. BMC Geriatrics. 2020;20(1):1-18. doi:10.1186/s12877-020-01801-7

20. Paddick SM, Gray WK, Ogunjimi L, et al. Validation of the Identification and Intervention for Dementia in Elderly Africans (IDEA) cognitive screen in Nigeria and Tanzania. BMC Geriatrics. 2015;15(1):1-9. doi:10.1186/s12877-015-0040-1

21. Bowles M, Bramhall J, Couch D, et al. Eligibility Framework for Scoring Support Levels.; 2017. https://www.natsip.org.uk/doc-library-login/eligibility-framework/00-eligibility-framework-summer-2017edition/916-eligibility-framework-document/file

22. ICD-10 Glaucoma Reference Guide; 2019.

23. Feroze K, Gulick P. HIV Retinopathy. StatPearls Publishing; 2019. https://www.ncbi.nlm.nih.gov/books/NBK470530/

24. World Health Organization and Calouste Gulbenkian Foundation. Integrating the response to mental disorders and other chronic diseases in health care systems. Geneva, World Health Organization. Published online 2014:1-50. http://apps.who.int/iris/bitstream/10665/112830/1/9789241506793_eng.pdf?ua=1

25. Pascolini D, Mariotti SP. Global estimates of visual impairment: 2010. British Journal of Ophthalmology. 2012;96(5):614-618. doi:10.1136/bjophthalmol-2011-300539

26. Sommer A, Taylor HR, Ravilla TD, et al. Challenges of ophthalmic care in the developing world. JAMA Ophthalmology. Published online 2014. doi:10.1001/jamaophthalmol.2014.84

27. International Agency for the Prevention of Blindness. Human Resources for Health Strategy Consultation. Vol 126.; 2014. Accessed July 13, 2019. http://www.who.int/workforcealliance/media/news/2014/7InternationalAgencyPreventionBlindness.pdf 28. Betjeman TJ, Soghoian SE, Foran MP. mHealth in Sub-Saharan Africa. 2013;2013. 
29. Resnikoff S, Lansingh VC, Washburn L, et al. Estimated number of ophthalmologists worldwide (International Council of Ophthalmology update): will we meet the needs? British Journal of Ophthalmology. 2020;104(4):588 LP - 592. doi:10.1136/bjophthalmol-2019-314336

30. Otiti-Sengeri J, Colebunders R, Kempen JH, Ronald A, Sande M, Katabira E. The prevalence and causes of visual loss among HIV-infected individuals in Uganda. Journal of Acquired Immune Deficiency Syndromes. 2010;53(1):95-101. doi:10.1097/QAl.0b013e3181c313f0

31. Mathenge W, Bastawrous A, Foster A, Kuper H. The Nakuru posterior segment eye disease study: Methods and prevalence of blindness and visual impairment in Nakuru, Kenya. Ophthalmology. Published online 2012. doi:10.1016/j.ophtha.2012.04.019

32. Lewallen S, Roberts $\mathrm{H}$, Hall A, et al. Increasing cataract surgery to meet Vision 2020 targets; experience from two rural programmes in east Africa. British Journal of Ophthalmology. 2005;89(10):1237-1240. doi:10.1136/bjo.2005.068791

33. Ivy A, Tam J, Dewhurst MJ, et al. Ambulatory blood pressure monitoring to assess the white-coat effect in an elderly east african population. Journal of Clinical Hypertension. 2015;17(5):389-394. doi:10.1111/jch. 12501

\section{Figures}




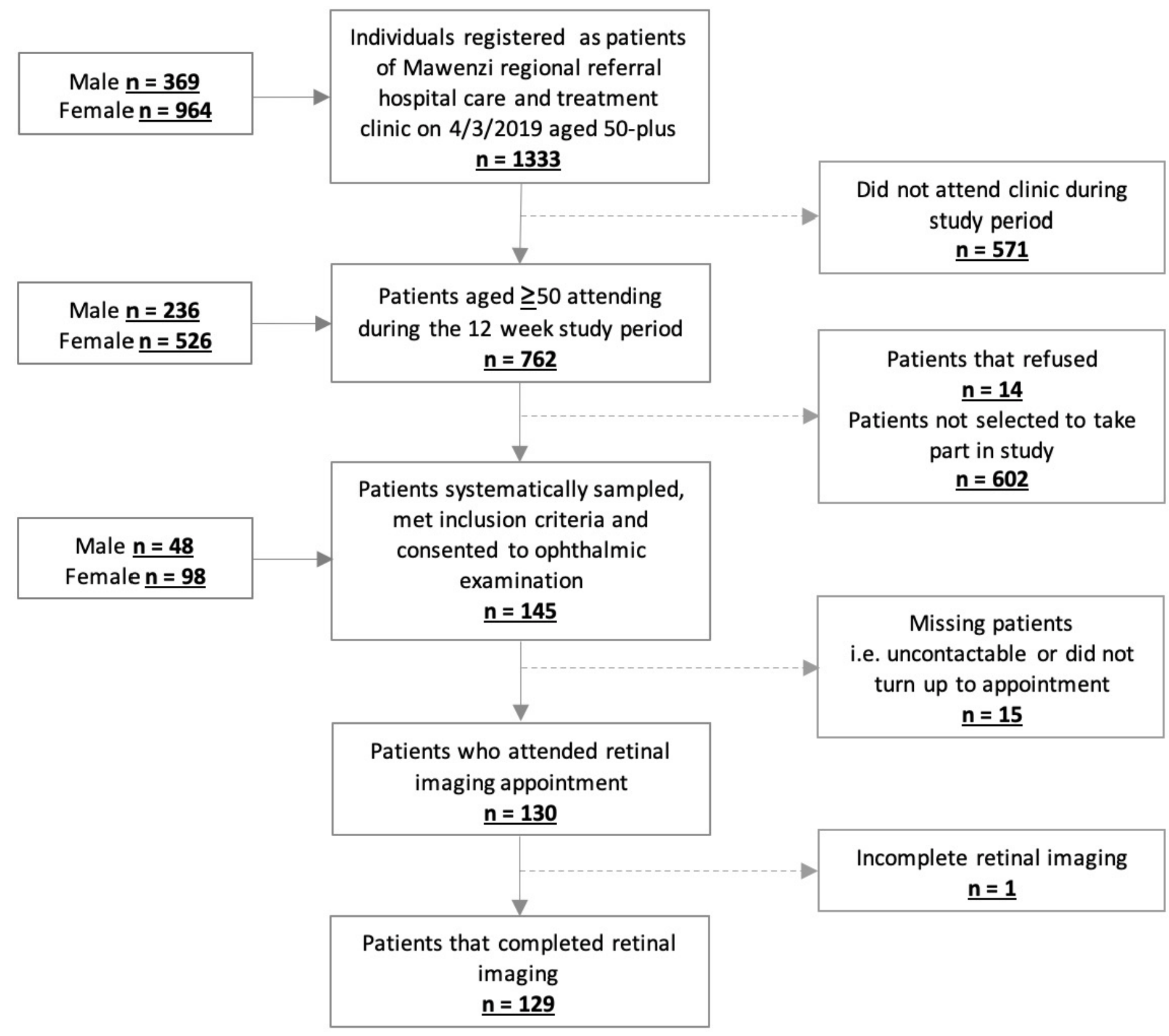

Figure 1

Of the 762 individuals aged $\geq 50$ years attending follow-up during the study period, 145 were systematically sampled and consented to inclusion. Of these, 129 completed the ophthalmologic assessment and had complete data (Figure 1). 